\title{
The Association between Meteorological Factors and the Prevalence of Acute-on-chronic Liver Failure: A Population-based Study, 2007-2016
}

\author{
Su Lin ${ }^{1}$, Lifen Han ${ }^{2}$, Dongliang Li ${ }^{3}$, Ting Wang ${ }^{1}$, Zimu Wu ${ }^{1}$, Haoyang Zhang ${ }^{4}$, Zhansong Xiao ${ }^{5}$, \\ Yinlian $\mathrm{Wu}^{1}$, Jiaofeng Huang ${ }^{1}$, Mingfang Wang ${ }^{1}$ and Yueyong Zhu*1 \\ ${ }^{1}$ Liver Research Center of the First Affiliated Hospital of Fujian Medical University, Fuzhou, Fujian, China; ${ }^{2}$ Department of \\ Infectious Disease, Mengchao Hepatobiliary Hospital of Fujian Medical University, Fuzhou, Fujian, China; ${ }^{3}$ Department of \\ Hepatobiliary Disease, 900 Hospital of PLA, Fuzhou, Fujian, China; ${ }^{4}$ School of Public Health, Sun Yat-sen University, Guangzhou, \\ Guangdong, China; ${ }^{5}$ Fujian Medical University, Fuzhou, Fujian, China
}

\section{Abstract}

Background and Aims: The aim of this study was to investigate the effect(s) of meteorological factors on the prevalence of acute-on-chronic liver failure (ACLF) based on 10-years' worth of population data. Methods: We retrospectively collected ACLF case data from January 2007 to December 2016 from three major hospitals in Fuzhou City, China. Climatic data, including rainfall, mean temperature, differences in temperature (delta temperature) and mean humidity for each month were downloaded from the China Climatic Data Service Center. Following data collection, Poisson regression analysis was used to estimate the effect(s) of climatic factors on the risk of the prevalence of ACLF. Results: The population consisted of a total of 3510 cases, with a mean age of $44.7 \pm$ 14.8 years-old and with $79.8 \%$ being male. Upon analyzing the population data, we found a growing trend and seasonal pattern of monthly counts of ACLF-related hospitalization throughout the past decade. Specifically, the primary peak of ACLF prevalence was in January and the secondary peak was in July. Poisson regression showed mean temperature (risk ratio $=0.991,95 \% \mathrm{CI}=0.986-0.996$ ) and mean humidity (risk ratio $=1.011,95 \% \mathrm{CI}=1.006-1.017$ ) to be independently correlated with the monthly cases of ACLF. The results suggest that every unit increase of mean temperature $\left(1^{\circ} \mathrm{C}\right)$ and mean humidity $(1 \%)$ are associated with 0.991 - and 1.011 -fold changes of ACLF cases, respectively. Rainfall and delta temperature did not appear to affect the prevalence of this disease. Conclusions: The hospitalization for ACLF peaks in January and July. Low temperature and high humidity appear to function as factors contributing to this seasonal pattern.

Citation of this article: Lin S, Han L, Li D, Wang T, Wu Z, Zhang $\mathrm{H}$, et al. The association between meteorological factors and the prevalence of acute-on-chronic liver failure: A population-based study, 2007-2016. J Clin Transl Hepatol 2019;7(4):341-345. doi: 10.14218/JCTH.2019.00044.

Keywords: Acute-on-chronic liver failure; Seasonal variation; Temperature; Humidity.

Abbreviations: ACLF, acute-on-chronic liver failure; CI, confidential interval; $\mathrm{HBV}$, hepatitis B virus.

Received: 6 September 2019; Revised: 13 November 2019; Accepted: 6 December 2019

*Correspondence to: Yueyong Zhu, Department of Liver Research Center, First Affiliated Hospital of Fujian Medical University, Fuzhou, Fujian 350001, China. Tel: +86-591-87981656, Fax: +86-591-87982526, E-mail: zhuyueyong@fjmu.edu.cn

\section{Introduction}

Acute-on-chronic liver failure (ACLF) is a critical clinical syndrome characterized by rapid development of hepatocellular dysfunction. ${ }^{1,2}$ Epidemiological and clinical data show that although the prevalence of ACLF is relatively low, it has an extremely poor survival rate (28-day mortality rate of 30$40 \%) \cdot{ }^{2-4}$ A widely accepted concept is that ACLF results from the acute deterioration of preexisting chronic liver diseases. ${ }^{5}$ Bacterial infection, ${ }^{6}$ viral infection, ${ }^{7,8}$ and alcohol intake ${ }^{9}$ have been shown to contribute to the initiation of ACLF. However, there are still $40 \%$ of patients with ACLF whose precipitating factors remain unknown. ${ }^{10}$

Meteorological factors, such as temperature, humidity and air pollution, can affect the health of human beings. Previous studies have proven the existence of seasonal variation in several critical diseases, and climatic factors are considered to play an important role in these seasonal patterns. ${ }^{11-16}$ In accordance with this knowledge, we also noticed that the number of patients hospitalized for ACLF has varied over time. However, we found few studies that focused on the effect of climatic factors on the risk of ACLF. Therefore, we developed this study to explore the seasonal patterns contributing to ACLF risk in a provincial city in Southeastern China and to identify the possible meteorological risk factors of ACLF based on 10-years' worth of population data. The results of our study may provide clues as to the underlying mechanisms that condition patterns of ACLF hospitalizations and may contribute to the health and medical fields by pointing to ways in which ACLF-related interventions might be better tailored in public health.

\section{Methods}

\section{Study population}

Hospitalization data for patients with ACLF as the main diagnosis were collected from administrative databases in three tertiary hospitals located in Fuzhou City, Fujian Province, China from January 2007 to December 2016. The hospitals included in this study were the First Affiliated Hospital of Fujian Medical University, the Mengchao Hepatobiliary Hospital of Fujian Medical University and the Fuzhou PLA General Hospital. Liver failure is an extremely dangerous 
clinical syndrome which requires immediate hospitalization, and only a limited number of hospitals in Fuzhou City are qualified to admit such patients. Therefore, approximately $90 \%$ of ACLF patients were cared for in the three hospitals mentioned listed above.

According to the International Classification of Diseases Version 10 (ICD 10), we used K70.4, K71.1, K72.0, K72.1 and $\mathrm{K} 72.9$ to identify cases of liver failure. Electronic records were manually reviewed by a research assistant (TW) and ACLF patients were selected based on diagnostic criteria from the Asian Pacific Association for the Study of the Liver (2014). ${ }^{17}$ The data we collected included the number of patients admitted every month, and each patient's admission date, age, sex, and detailed diagnosis. In cases where the patient was being transferred among any of the three above-mentioned hospitals, the earliest hospitalization data was used for that particular patient.

\section{Meteorological data}

Fuzhou City, with an area of $12,177 \mathrm{~km}^{2}$ and a population of 6.78 million as of 2015 , is located on the Southeast coast of Fujian Province, which is set in the Southeastern region of China (see data from National Bureau of Statistics of the People's Republic of China, http://www.stats.gov.cn/). Fuzhou City has a typical subtropical climate, in which the summers are long, hot and humid, while the winters are short, mild and less rainy. The average temperature ranges from $10.9^{\circ} \mathrm{C}$ in January to $28.9^{\circ} \mathrm{C}$ in July. The annual mean temperature is $19.84^{\circ} \mathrm{C}$. The meteorological data, including rainfall (in milliliters), average temperature $\left({ }^{\circ} \mathrm{C}\right)$, the difference between the monthly highest temperature and lowest temperature (delta-temperature, ${ }^{\circ} \mathrm{C}$ ) and average humidity (\%) of each month, were derived from the China Climatic Data Service Center (http://data.cma.cn/, station number: 58847).

\section{Statistical analyses}

To explore the trends and seasonal patterns of monthly ACLF cases, an automatic exponential model with trends and seasonal components was used to fit the time series of ACLF cases. Univariate and multivariate Poisson regressions were used to measure the effect of climatic factors on ACLF prevalence. Statistical analysis was performed using R software (version 3.3.2, https://cran.r-project.org). All tests were two-sided and $p$-value $<0.05$ was considered statistically significant.

\section{Ethical statement}

This study was approved by the Ethical Review Committee of the First Affiliated Hospital of Fujian Medical University (Permission number: FYDYFY-0014211). All methods were performed in accordance with the Declaration of Helsinki, regarding ethical standards for research involving human subjects.

\section{Patient and public involvement}

No patient was involved in the design of this study, including in the development of the research question and outcome measures, in the recruitment and conduct of the study, nor in the final manuscript writing. The results will not be disseminated to study participants.

\section{Results}

\section{Demographic characteristics of 3510 patients with ACLF}

A total of 3510 patients with ACLF were identified from 20072016 , with $2593(73.8 \%)$ cases of hepatitis B virus (HBV) infection, $158(4.5 \%)$ cases of alcoholic hepatitis, $213(6.4 \%)$ cases who had both the above risk factors, and $546(15.6 \%)$ cases complicated with other etiologies (including hepatitis A or hepatitis $\mathrm{E}$ infection in patients with underlying cirrhosis, autoimmune hepatitis, Wilson's disease, drug-induced hepatitis, and ACLF with unknown etiologies). HBV-related ACLF constituted the majority of this cohort. The mean age of this population was $44.7 \pm 14.8$ years-old and $79.8 \%$ of the patients were male (Table 1 ). The distribution of different etiologies of ACLF from each year is shown in Fig. 1 and Table 1.

\section{Seasonal variance of ACLF}

The seasonal variance of ACLF over 10 years is shown in Fig. 2. The time series of ACLF was divided into seasonal components, trend components, and irregular components. Although ACLF occurred throughout the year, the cold season was associated with a higher disease prevalence. This seasonal effect suggested the highest peak of ACLF was in January, which was followed by a smaller peak in July. No significant difference in seasonal pattern was found between the total ACLF cases and HBV-ACLF.

\section{Association between the prevalence of ACLF and climatic factors}

Four major climatic factors were explored, including rainfall (in milliliters), mean monthly temperature $\left({ }^{\circ} \mathrm{C}\right)$, delta temperature, and mean humidity (\%). Univariate analysis showed that rainfall was not associated with the prevalence of $A C L F$, but that mean temperature and delta temperature were negatively related to ACLF, while humidity was positively associated with ACLF cases (Table 2). After including these three variables in a multivariate analysis, only mean temperature (risk ratio $=0.991,95 \%$ confidential interval $(\mathrm{CI})=$ $0.986-0.996$ ) and mean humidity (risk ratio $=1.011,95 \%$

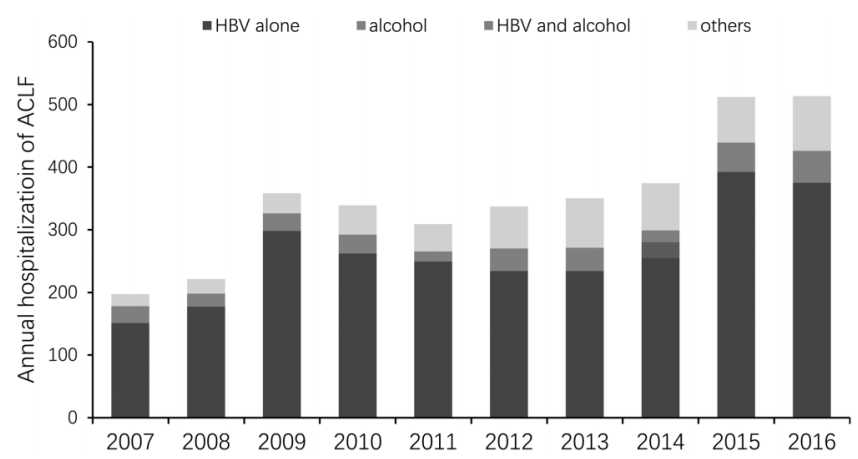

Fig. 1. Distribution of different etiologies of ACLF in each year.

Abbreviation: ACLF, acute-on-chronic liver failure. 
Lin S. et al: Meteorological factors and ACLF

Table 1. Demographic characteristics of 3510 ACLF patients

\begin{tabular}{|c|c|c|c|c|}
\hline Characteristics & $\begin{array}{l}\text { Overall, } \\
n=3510\end{array}$ & $\begin{array}{l}\text { First Affiliated } \\
\text { Hospital, } n=823\end{array}$ & $\begin{array}{l}\text { Fuzhou PLA General } \\
\text { Hospital, } n=697\end{array}$ & $\begin{array}{l}\text { Mengchao Hepatobiliary } \\
\text { Hospital, } n=1990\end{array}$ \\
\hline Age (years), mean $\pm S D$ & $44.7 \pm 14.8$ & $46.5 \pm 14.9$ & $46.9 \pm 15.6$ & $42.5 \pm 14.0$ \\
\hline Male, $n(\%)$ & $2802(79.8 \%)$ & $638(77.5 \%)$ & $533(76.4 \%)$ & $1631(82.0 \%)$ \\
\hline \multicolumn{5}{|l|}{ Etiology, $n(\%)$} \\
\hline HBV alone & $2593(73.8 \%)$ & $611(74.2 \%)$ & $407(58.4 \%)$ & $1575(79.1 \%)$ \\
\hline Alcohol alone & $158(4.5 \%)$ & $56(6.8 \%)$ & $40(5.8 \%)$ & $62(3.1 \%)$ \\
\hline HBV \& alcohol & $213(6.1 \%)$ & $30(3.6 \%)$ & $28(4.0 \%)$ & $155(7.8 \%)$ \\
\hline Others & $546(15.6 \%)$ & $126(15.3 \%)$ & $222(31.9 \%)$ & $198(9.9 \%)$ \\
\hline \multicolumn{5}{|l|}{ Years } \\
\hline 2007 & $197(5.6 \%)$ & $62(7.5 \%)$ & $56(8.0 \%)$ & $79(4.0 \%)$ \\
\hline 2008 & $221(6.3 \%)$ & $75(9.1 \%)$ & $55(7.9 \%)$ & $91(4.6 \%)$ \\
\hline 2009 & $358(10.2 \%)$ & $79(9.6 \%)$ & $56(8.0 \%)$ & $223(11.2 \%)$ \\
\hline 2010 & $339(9.7 \%)$ & $74(9.0 \%)$ & $74(10.6 \%)$ & $191(9.6 \%)$ \\
\hline 2011 & $309(8.8 \%)$ & $83(10.1 \%)$ & $56(8.0 \%)$ & $170(8.5 \%)$ \\
\hline 2012 & 337 (9.6\%) & $102(12.4 \%)$ & $50(7.2 \%)$ & $185(9.3 \%)$ \\
\hline 2013 & 349 (9.9\%) & $82(10.0 \%)$ & $84(12.1 \%)$ & $183(9.2 \%)$ \\
\hline 2014 & $375(10.7 \%)$ & $85(10.3 \%)$ & $84(12.1 \%)$ & $206(10.4 \%)$ \\
\hline 2015 & $512(14.6 \%)$ & $89(10.8 \%)$ & $87(12.5 \%)$ & $336(16.9 \%)$ \\
\hline 2016 & $513(14.6 \%)$ & $92(11.2 \%)$ & 95 (13.6\%) & $326(16.4 \%)$ \\
\hline
\end{tabular}

Abbreviations: ACLF, acute-on-chronic liver failure; HBV, hepatitis B virus; SD, standard deviation.

$\mathrm{CI}=1.006-1.017)$ were found to be independently correlated with the prevalence of ACLF cases. The results suggested that every unit increase of mean temperature $\left(1^{\circ} \mathrm{C}\right)$ and mean humidity ( $1 \%$ ) were associated with 0.991 - and 1.011 -fold changes in ACLF cases, respectively.

Smoothing plots of monthly mean temperature and mean relative humidity in relation to ACLF cases are shown in Fig. 3. Despite some fluctuations of the curve, it can be clearly seen that the risk of ACLF increased with mean humidity. The peak of the relative risk of ACLF was at a mean temperature of $12^{\circ}$ $C$, which was the average temperature of Fuzhou City in January. The lowest risk of ACLF was associated with the humidity of $61 \%$ and a temperature of $16^{\circ} \mathrm{C}$.

\section{Discussion}

ACLF has raised an increasing concern because of its high mortality rate and heavy disease burden. ${ }^{3,9,18}$ A growing trend in the incidence of this fatal disease throughout the past decade has been observed in the USA. ${ }^{2,18}$ In this study, although a different diagnostic criterion for ACLF was used, we also demonstrated a rise in the prevalence of ACLF in Southeastern China. Additionally, a correlation between the prevalence of ACLF and low temperature as well as high humidity was demonstrated in this study.

The results showed that, although ACLF occurs throughout the year, cold seasons are associated with higher prevalence. Seasonal variance has also been found in less severe liver diseases. This pattern seems to be dependent on the etiology of liver diseases: hepatitis $A$ and hepatitis $E$ have prominent peaks in the spring and summer, ${ }^{19}$ primary biliary cirrhosis peaks in June, ${ }^{20}$ and hepatitis $B$ peaks in January and Febru- ary. ${ }^{21}$ Similarly, this study found seasonal variance associated with the incidence of ACLF, a fatal liver disease. These results revealed again the existence of seasonal pattern of liver diseases.

We found the number of ACLF hospital admissions peaked in winter. The winter season has frequently been shown to increase the risks of critical diseases. ${ }^{11-14}$ Part of the reason for this could be related to lower temperatures since low environmental temperatures induce immunosuppression in vertebrates. In response, animals have to increase their immune activities to maintain immune balance. ${ }^{22}$ Proinflammatory factors' expressions as well as macrophage and T-cell infiltration are up-regulated in animals after cold exposure, causing systemic inflammation in lower temperatures. Given that excessive systemic inflammation has been known to play a crucial role in the development of $\mathrm{ACLF}^{10}$ changes in the immune system due to low temperature may also contribute to its seasonality.

From a different perspective, it is also the case that the decreased sun exposure in winter which has been shown to be associated with higher probabilities of vitamin D deficiency. ${ }^{23}$ Low vitamin D serum concentration significantly correlates with liver diseases, including nonalcoholic fatty liver disease ${ }^{24}$ and hepatitis $B \cdot{ }^{25,26}$ Vitamin D deficiency leads to higher levels of $\mathrm{HBV}$ replication, which increases the risk of infection and often leads to poor outcomes in patients with liver cirrhosis. ${ }^{25,26}$ Moreover, temperature and light have also been shown to play a role in regulating DNA methylation. ${ }^{27}$ Fitting for our study, it is also shown that aberrant GSTP1 promoter methylation is associated with the development of ACLF and its prognosis. ${ }^{28}$ These factors, especially when they are combined, might help explain why ACLF peaks in winter. However, further research is 
Total ACLF

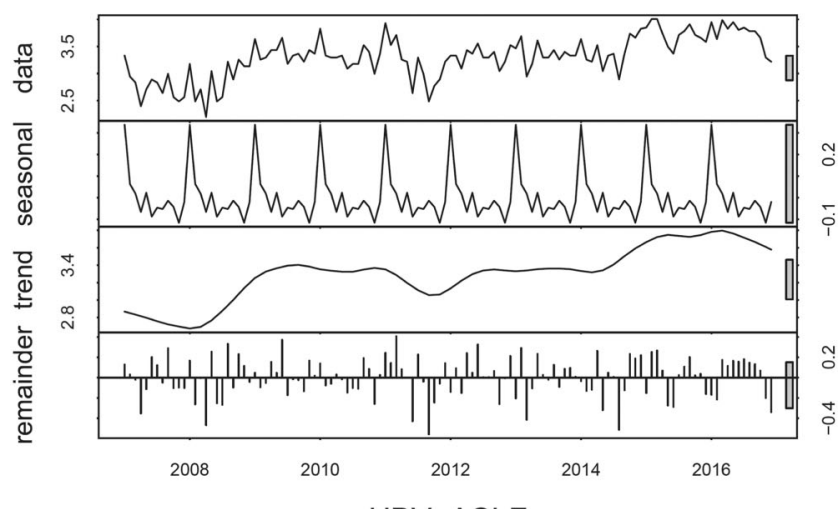

HBV-ACLF

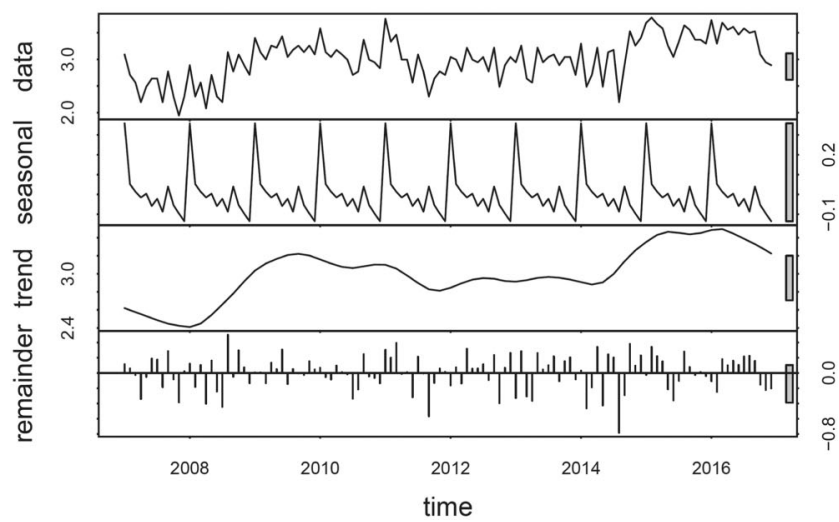

Fig. 2. The seasonal variations of ACLF over $\mathbf{1 0}$ years. The time series is divided into seasonal, trend, and irregular components. The total ACLF is demonstrated on the upper panel and HBV-ACLF is shown on the lower panel. The grey bars on the right represent the same magnitude. The time trend of ACLF prevalence is shown to have been increasing over the last decade. The seasonal effect suggests the peak prevalence of ACLF is in January. No difference in seasonal pattern is found between the total ACLF and HBV-ACLF.

Abbreviations: ACLF, acute-on-chronic liver failure; $\mathrm{HBV}$, hepatitis $\mathrm{B}$ virus.

still needed to elucidate the mechanistic links between temperature and ACLF.

Our study found a positive relationship between high humidity and the prevalence of ACLF, which is not surprising considering that moisture environment accelerates bacteria growth ${ }^{15}$ and that warm-humid conditions increase the risk of infection. ${ }^{29}$ Infection is one of the most important precipitating factors of ACLF. 6,30,31 Therefore, humidity likely has an indirect effect on ACLF onset and high humidity might also
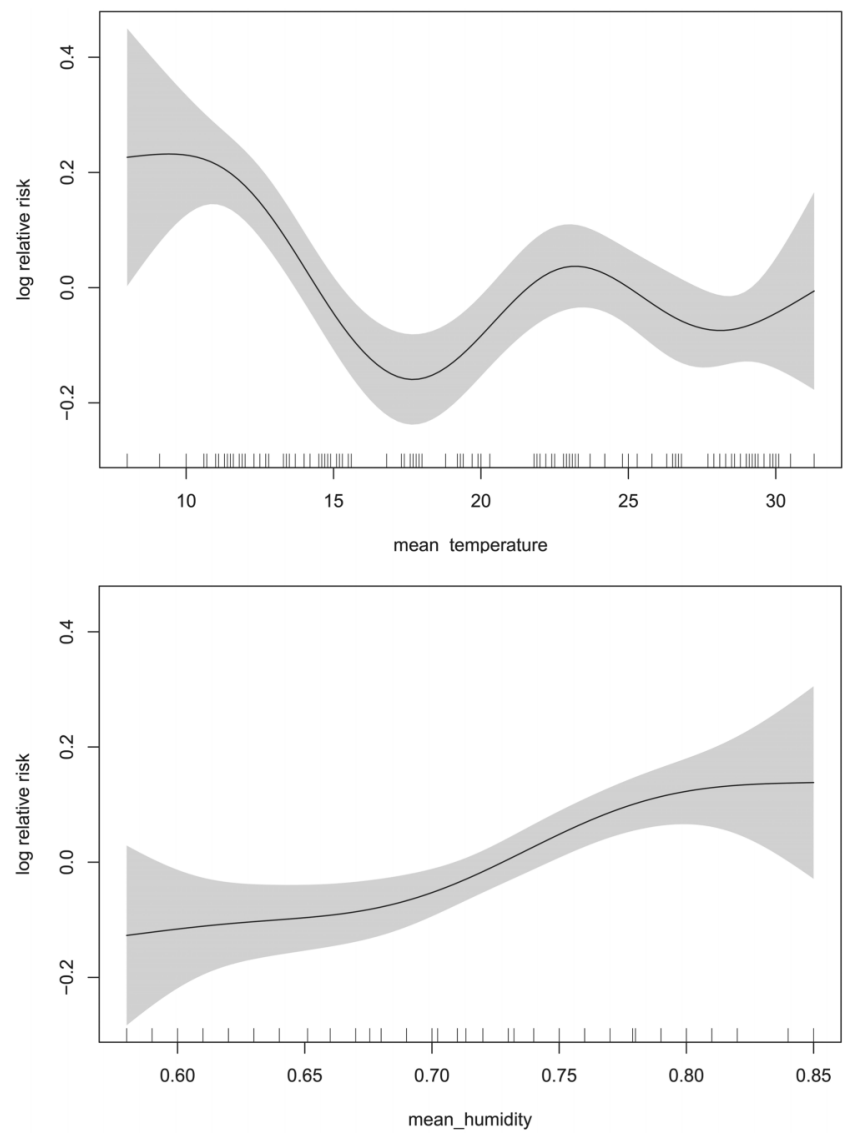

Fig. 3. Smoothing plots of monthly mean temperature and mean relative humidity against ACLF. The risk of ACLF can be seen to have increased with higher humidity and lower temperature. The peak of the relative risk of ACLF was at a temperature of $12^{\circ} \mathrm{C}$, which was the average temperature of Fuzhou City in January.

Abbreviation: ACLF, acute-on-chronic liver failure.

explain the small peak of ACLF in July, as we found in our cohort study.

The major advantage of this study is that it is the first population-based study to investigate the correlation between climatic factors and ACLF risk. Even so, it also has limitations. The major weakness of this study is that we only evaluated the condition of a single city. Therefore, the results of our study still need to be verified in other regions. Furthermore, we only focused on the climate factors that influence ACLF onset; however, there are various confounding factors which may have impacted this liver disease, such as

Table 2. Poisson regression for the risk of ACLF

\begin{tabular}{|c|c|c|c|c|c|c|}
\hline \multirow[b]{2}{*}{ Meteorological factor } & \multicolumn{3}{|l|}{ Univariate } & \multicolumn{3}{|l|}{ Multivariate } \\
\hline & Risk ratio & $95 \% \mathrm{CI}$ & $p$ & Risk ratio & $95 \% \mathrm{CI}$ & $p$ \\
\hline Mean temperature in ${ }^{\circ} \mathrm{C}$ & 0.991 & $0.986-0.996$ & $<0.001$ & 0.991 & $0.986-0.996$ & 0.001 \\
\hline Mean humidity, \% & 1.012 & $1.007-1.018$ & $<0.001$ & 1.011 & $1.006-1.017$ & $<0.001$ \\
\hline Delta temperature in ${ }^{\circ} \mathrm{C}$ & 0.945 & $0.920-0.979$ & $<0.001$ & 0.973 & $0.945-1.002$ & 0.066 \\
\hline Rainfall in milliliter & 1 & $1-1$ & 0.719 & & & \\
\hline
\end{tabular}

Abbreviation: ACLF, acute-on-chronic liver failure. 
alcohol consumption or upper respiratory infections. These factors are prevalent in winter and summer as well, but it is difficult to collect such information in a retrospective study. Additionally, well-designed, prospective research is required to address these issues.

\section{Conclusions}

A seasonal variation exists in ACLF hospitalization, which peaks in January and July. Low temperature and high humidity appear to be the primary contributing factors to this seasonal pattern.

\section{Acknowledgments}

This study was funded by Fujian Provincial Health Technology Project (2016-CX-33), Fujian Science and Technology Project (2017j01187), Fujian Province Science and Technology Program (2019D002) and Fuzhou Science and Technology Program (2018-S-103-1). They supported the analysis of the data and writing of the manuscript.

\section{Conflict of interest}

The authors have no conflict of interests related to this publication.

\section{Author contributions}

Designed the study ( $S L$ and $Y Z$ ), collected and cleaned the data (TW, HL, DL, YW, JH, ZX and MW), analyzed the data (SL, $\mathrm{HZ}$ and $\mathrm{ZW}$ ), wrote the manuscript (SL), contributed to supervising the project and checked the final version of the manuscript $(\mathrm{YZ})$. All authors read and approved the final version of the manuscript.

\section{References}

[1] Hernaez R, Solà E, Moreau R, Ginès P. Acute-on-chronic liver failure: an update. Gut 2017;66:541-553. doi: 10.1136/gutjnl-2016-312670.

[2] Allen AM, Kim WR, Moriarty JP, Shah ND, Larson JJ, Kamath PS. Time trends in the health care burden and mortality of acute on chronic liver failure in the United States. Hepatology 2016;64:2165-2172. doi: 10.1002/hep.28812.

[3] Allen AM, Kim WR. Epidemiology and healthcare burden of acute-on-chronic liver failure. Semin Liver Dis 2016;36:123-126. doi: 10.1055/s-00361583201.

[4] Lin S, Zhang K, Zhang J, Wang M, Velani B, Zhu Y. Long-term outcomes of patients with hepatitis $B$ virus-related acute on chronic liver failure: An observational cohort study. Liver Int 2019;39:854-860. doi: 10.1111/liv. 14072.

[5] Anand AC, Dhiman RK. Acute on chronic liver failure-what is in a 'definition'? J Clin Exp Hepatol 2016;6:233-240. doi: 10.1016/j.jceh.2016.08.011.

[6] Fernández J, Acevedo J, Wiest R, Gustot T, Amoros A, Deulofeu C, et al. Bacterial and fungal infections in acute-on-chronic liver failure: prevalence, characteristics and impact on prognosis. Gut 2018;67:1870-1880. doi: 10. 1136/gutjnl-2017-314240.

[7] Frias M, López-López P, Rivero A, Rivero-Juarez A. Role of hepatitis E virus infection in acute-on-chronic liver failure. Biomed Res Int 2018;2018: 9098535. doi: 10.1155/2018/9098535.

[8] Cao Z, Liu Y, Wang S, Lu X, Yin S, Jiang S, et al. The impact of HBV flare on the outcome of HBV-related decompensated cirrhosis patients with bacterial infection. Liver Int 2019;39:1943-1953. doi: 10.1111/liv.14176.

[9] Singal AK, Arora S, Wong RJ, Satapathy SK, Shah VH, Kuo YF, et al. Increasing burden of acute-on-chronic liver failure among alcohol-associated liver disease in the young population in the United States. Am J Gastroenterol 2019. doi: 10.14309/ajg.0000000000000411.

[10] Clària J, Stauber RE, Coenraad MJ, Moreau R, Jalan R, Pavesi M, et al. Systemic inflammation in decompensated cirrhosis: Characterization and role in acute-on-chronic liver failure. Hepatology 2016;64:1249-1264. doi: 10. 1002/hep.28740.

[11] Cheng J, Xu Z, Bambrick H, Su H, Tong S, Hu W. Impacts of heat, cold, and temperature variability on mortality in Australia, 2000-2009. Sci Total Environ 2019;651:2558-2565. doi: 10.1016/j.scitotenv.2018.10.186.

[12] Sun Z, Chen C, Xu D, Li T. Effects of ambient temperature on myocardial infarction: A systematic review and meta-analysis. Environ Pollut 2018; 241:1106-1114. doi: 10.1016/j.envpol.2018.06.045.

[13] Morral-Puigmal C, Martínez-Solanas Ė, Villanueva CM, Basagaña X. Weather and gastrointestinal disease in Spain: A retrospective time series regression study. Environ Int 2018;121:649-657. doi: 10.1016/j.envint.2018.10.003.

[14] Chen R, Yin P, Wang L, Liu C, Niu Y, Wang W, et al. Association between ambient temperature and mortality risk and burden: time series study in 272 main Chinese cities. BMJ 2018;363:k4306. doi: 10.1136/bmj.k4306.

[15] Redfern J, Verran J. Effect of humidity and temperature on the survival of Listeria monocytogenes on surfaces. Lett Appl Microbiol 2017;64:276-282. doi: $10.1111 /$ lam.12714.

[16] Kim BI, Ki H, Park S, Cho E, Chun BC. Effect of climatic factors on hand, foot, and mouth disease in South Korea, 2010-2013. PLoS One 2016;11: e0157500. doi: 10.1371/journal.pone.0157500.

[17] Sarin SK, Kedarisetty CK, Abbas Z, Amarapurkar D, Bihari C, Chan AC, et al. Acute-on-chronic liver failure: consensus recommendations of the Asian Pacific Association for the Study of the Liver (APASL) 2014. Hepatol Int 2014;8:453-471. doi: 10.1007/s12072-014-9580-2.

[18] Kim D, Cholankeril G, Li AA, Kim W, Tighe SP, Hameed B, et al. Trends in hospitalizations for chronic liver disease-related liver failure in the United States, 2005-2014. Liver Int 2019;39:1661-1671. doi: 10.1111/liv.14135.

[19] Fares A. Seasonality of hepatitis: a review update. J Family Med Prim Care 2015;4:96-100. doi: 10.4103/2249-4863.152263.

[20] McNally RJ, James PW, Ducker S, James OF. Seasonal variation in the patient diagnosis of primary biliary cirrhosis: further evidence for an environmental component to etiology. Hepatology 2011;54:2099-2103. doi: 10.1002/hep. 24597.

[21] Gan R, Chen N, Huang D. Comparisons of forecasting for hepatitis in Guangxi Province, China by using three neural networks models. Peer] 2016;4: e2684. doi: 10.7717/peerj.2684.

[22] Martin LB, Weil ZM, Nelson RJ. Seasonal changes in vertebrate immune activity: mediation by physiological trade-offs. Philos Trans R Soc Lond B Biol Sci 2008;363:321-339. doi: 10.1098/rstb.2007.2142.

[23] Rosecrans R, Dohnal JC. Seasonal vitamin D changes and the impact on health risk assessment. Clin Biochem 2014;47:670-672. doi: 10.1016/j. clinbiochem.2014.02.004.

[24] Liu S, Liu Y, Wan B, Zhang H, Wu S, Zhu Z, et al. Association between vitamin D status and non-alcoholic fatty liver disease: A population-based study. J Nutr Sci Vitaminol (Tokyo) 2019;65:303-308. doi: 10.3177/jnsv.65.303.

[25] Farnik H, Bojunga J, Berger A, Allwinn R, Waidmann O, Kronenberger B, et al. Low vitamin $D$ serum concentration is associated with high levels of hepatitis B virus replication in chronically infected patients. Hepatology 2013;58: 1270-1276. doi: 10.1002/hep.26488.

[26] Konstantakis C, Tselekouni P, Kalafateli M, Triantos C. Vitamin D deficiency in patients with liver cirrhosis. Ann Gastroenterol 2016;29:297-306. doi: 10. 20524/aog.2016.0037.

[27] Alvarado S, Fernald RD, Storey KB, Szyf M. The dynamic nature of DNA methylation: a role in response to social and seasonal variation. Integr Comp Biol 2014;54:68-76. doi: 10.1093/icb/icu034.

[28] Gao S, Sun FK, Fan YC, Shi CH, Zhang ZH, Wang LY, et al. Aberrant GSTP1 promoter methylation predicts short-term prognosis in acute-on-chronic hepatitis B liver failure. Aliment Pharmacol Ther 2015;42:319-329. doi: 10.1111/apt.13271.

[29] Gao J, Sun Y, Lu Y, Li L. Impact of ambient humidity on child health: a systematic review. PLoS One 2014;9:e112508. doi: 10.1371/journal.pone. 0112508.

[30] Verma N, Singh S, Taneja S, Duseja A, Singh V, Dhiman RK, et al. Invasive fungal infections amongst patients with acute-on-chronic liver failure at high risk for fungal infections. Liver Int 2019;39:503-513. doi: 10.1111/liv. 13981.

[31] Mücke MM, Rumyantseva T, Mücke VT, Schwarzkopf K, Joshi S, Kempf VAJ, et al. Bacterial infection-triggered acute-on-chronic liver failure is associated with increased mortality. Liver Int 2018;38:645-653. doi: 10.1111/liv. 13568. 